\title{
Mitochondrial Toxicity of Drugs
}

\author{
Liliane Todesco, Katri Waldhauser, and Stephan Krähenbühl*
}

\begin{abstract}
Mitochondria are important targets of drug toxicity. A variety of drugs has been shown to affect the electron transport chain, coupling of oxidative phosphorylation, $\beta$-oxidation or other mitochondrial functions. Such damaging events may lead to the opening of a large pore across the mitochondrial membranes - the membrane permeability transition pore - eventually leading to apoptosis or necrosis of cells, depending on the cellular ATP content. Such drugs may therefore lead to organ damage, particularly in the liver, kidney, heart or skeletal muscle.
\end{abstract}

Keywords: Apoptosis · Drug toxicity · Mitochondria - Necrosis · $\beta$-Oxidation · Respiratory chain

\section{Introduction}

During the last years, mitochondrial damage has been recognized as one of the most important causes for adverse reactions of many drugs and toxins. The fact that mitochondria represent a target of drug toxicity is not surprising, since these organelles have a central function in cellular energy production, contain multiple metabolic pathways and are key players in the initiation of apoptosis and/or necrosis of a cell [1]. The mitochondrial respiratory chain, which is an important target of drug toxicity, is illustrated in Fig. 1.

As illustrated in the Table, drugs can affect mitochondria by a variety of mechanisms. One well-defined mechanism is inhibition of the electron flow across the electron transport chain (enzyme complexes I-IV, Fig. 1). The mechanisms by which these substances can impair electron flow within the electron transport chain include direct inhibition of a protein subunit of one (or more) of the enzyme complexes or acceptance of electrons flowing across the electron transport chain instead of the natural acceptors ubiquinone or cytochrome $\mathrm{c}$.

\footnotetext{
${ }^{*}$ Correspondence: S. Krähenbühl, MD, PhD Clinical Pharmacology \& Toxicology

University Hospital

$\mathrm{CH}-4031$ Basel

Tel.: +41612654715

Fax: +4161265 4560

E-Mail: kraehenbuehl@uhbs.ch
}

Uncoupling oxidative phosphorylation is another well-defined mechanism for mitochondrial toxicity. Uncoupling means that the protons, shifted from the mitochondrial matrix to the space between inner and outer membrane, do not pass across the $\mathrm{F}_{0} \mathrm{~F}_{1}$ ATPase (complex V) back to the mitochondrial matrix, but instead go directly across the inner mitochondrial membrane. The result is production of heat, but not of energy in the form of ATP. Typical examples of this mechanism include weak acids and weak bases [1], which can be protonated in

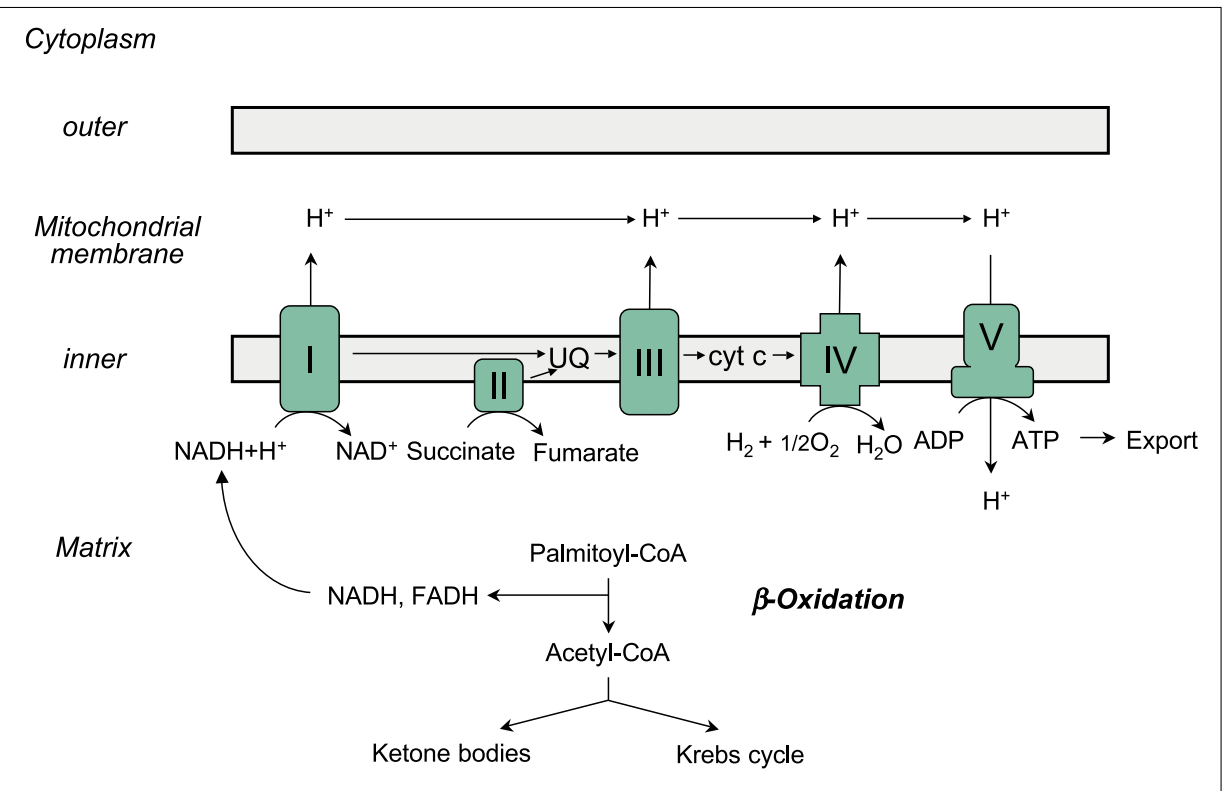

Fig. 1. Mitochondrial respiratory chain. NADH and FADH produced by $\beta$-oxidation and other metabolic pathways within the mitochondrial matrix are metabolized by the electron transport chain consisting of the enzyme complexes I, II, III and IV. Ubiquinol (UQ) and cytochrome c (cyt c) transport electrons between complexes I or II and III, and between complexes III and IV, respectively. Complexes I, III and IV can shift protons from the mitochondrial matrix into the inter-membrane space, building up a proton gradient. This gradient is necessary to produce ATP from ADP by complex $V$ or $F_{0} F_{1}$ ATPase. $\beta$-Oxidation also produces acetyl-CoA which can be used for the formation of ketone bodies, or is degraded to $\mathrm{CO}_{2}$ and $\mathrm{H}_{2} \mathrm{O}$ by the Krebs cycle. Further explanations are given in the text. 
Table. Mitochondrial toxicity of drugs: principle mechanisms and typical examples

- Inhibition of the electron transport chain Amiodarone, anthraline, benzbromarone, benzarone, buprenorphine, flutamide, MPP+, oxmetidine, perhexiline

- Uncoupling of oxidative phosphorylation Amiodarone, benzbromarone, benzarone, bupivacaine, buprenorphine, cerivastatin, etidocaine, tacrine

- Mitochondrial permeability transition Benzbromarone, benzarone, salicylate, valproate

- Inhibition of mitochondrial fatty acid metabolism

Amiodarone, asparaginase, benzbromarone, benzarone buprenorphine, female sex hormones, NSAIDs, salicylate, tetracycline, valproate

- Oxidation of mitochondrial DNA Alcohol

- Inhibition of mitochondrial DNA synthesis Nucleoside analogues, e.g. zidovudine, fialuridine

The final mechanism is damage to the mitochondrial DNA by oxidation or by inhibition of DNA synthesis [3]. Mitochondrial DNA is more susceptible to oxidative damage than nuclear DNA due to absence of histones and efficient repair mechanisms in mitochondria, and also due to the proximity of mitochondrial DNA to the oxygen radicals producing respiratory chain. A class of drugs known to potentially impair the synthesis of mtDNA are nucleoside analogues used in the treatment of HIV or hepatitis B infections.

Opening up a mega-channel across the mitochondrial membranes (membrane permeability transition pore), which leads to collapse of the membrane potential and swelling of the mitochondria, is another potential mechanism for drug toxicity. Opening this mega-channel can lead to mitochondrial loss of cytochrome $\mathrm{c}$, initiating the apoptosis cascade and eventually resulting in cell death [4].

\section{Our Studies in this Field}

\section{Valproate Hepatotoxicity}

Valproate, a medium-chain branched fatty acid, is used frequently in the treatment of patients with different forms of epilepsy. Very occasionally, the intake of valproate can be associated with fulminant hepatic failure and death [5]. The most probable mechanism of valproate-associated hepatotoxicity is the production of toxic metabolites such as 4-ene-valproate, a toxin inhibiting mitochondrial $\beta$-oxidation [6] (see Fig. 2 for valproate metabolism). Other mechanisms may contribute, in particular depletion of the hepatocellular free coenzyme A pool, and the formation of hepatotoxic acyl-CoAs [7]. We have investigated the CoA and carnitine metabolites in a liver from a patient with valproate-associated fulminant liver failure, and could demonstrate the expected decrease in the hepatic coenzyme A content and alterations in the hepatic carnitine pool [7].

The hallmark of liver histology in such patients is microvesicular steatosis, reflecting inhibition of mitochondrial $\beta$-oxidation [5][6]. Since not all patients treated with valproate develop hepatotoxicity, accumulation of 4-ene-valproate alone is not enough to explain the hepatotoxicity associated with this drug. In the search for additional risk factors, we investigated the siblings of the index patients with fulminant liver failure reported above [8]. The sister of the index patient had died from respiratory failure due to a mitochondrial disorder. In addition, her brother and mother also had clinical signs of a mitochondrial disorder, which could not be defined in more detail. Accordingly, in a muscle biopsy of the sister of the index patient, complexes I and IV of the respiratory chain showed a reduced activity, but the mitochondrial genome did not show mutations. The presence of a mitochondrial disorder, which may be sub-clinical, can therefore be regarded as a risk factor for the development of fulminant liver failure in patients treated with valproate. We are currently investigating this possibility in animal models with impaired hepatic mitochondrial $\beta$-oxidation.

In addition, treatment with valproate is associated with the formation of a variety of acylcarnitines, and with a decreased carnitine plasma concentration [9]. Since we have developed a method for the determination of carnitine and acylcarnitines in plasma by LC/ MS [10], we intend to study the acylcarnitines accumulating in the plasma of patients treated with valproate. Furthermore, it will be interesting to investigate the interaction between these acylcarnitines and carnitine on the level of OCTN2, the carnitine transporter responsible for proximal tubular reabsorption of carnitine [11]. We have stably expressed OCTN2 in different cell lines, and are going to use this system to study the transport of carnitine in the presence of the acylcarnitines detected in the plasma and urine of patients treated with valproate. Since our LC/MS method also allows the determination of butyrobetaine, the direct precursor of carnitine, we will be able to study the effect of valproate on renal clearance of butyrobetaine.

\section{Amiodarone}

Amiodarone is a class III antiarrhythmic used widely to treat cardiac arrhythmias particularly in patients with coronary heart disease. In patients and animals, amiodarone can be associated with hepatocellular injury, which may be due to liver steatosis [12]. In mice and rats, it has been shown that amiodarone uncouples oxidative phosphorylation, and inhibits $\beta$-oxidation of liver mitochondria [13][14].

Regarding amiodarone, we were interested to find out the structure which is finally responsible for its mitochondrial toxicity, and whether it is possible to obtain a pharmacologically active amiodarone derivative (block of hERG channels) without mitochondrial toxicity. In a first study, we confirmed amiodarone's mitochondrial toxicity in rats, and could demonstrate the importance of the configuration of the diethylaminoethoxy side chain for its mitochondrial toxicity [15]. These data allowed us to synthesize additional derivatives with alterations in the side chain, rendering it more hydrophilic (Fig. 3). Indeed, we were able to detect two derivatives, which demonstrated almost no mitochondrial toxicity. These derivatives contain only an ethoxy or an acetoxy group in the

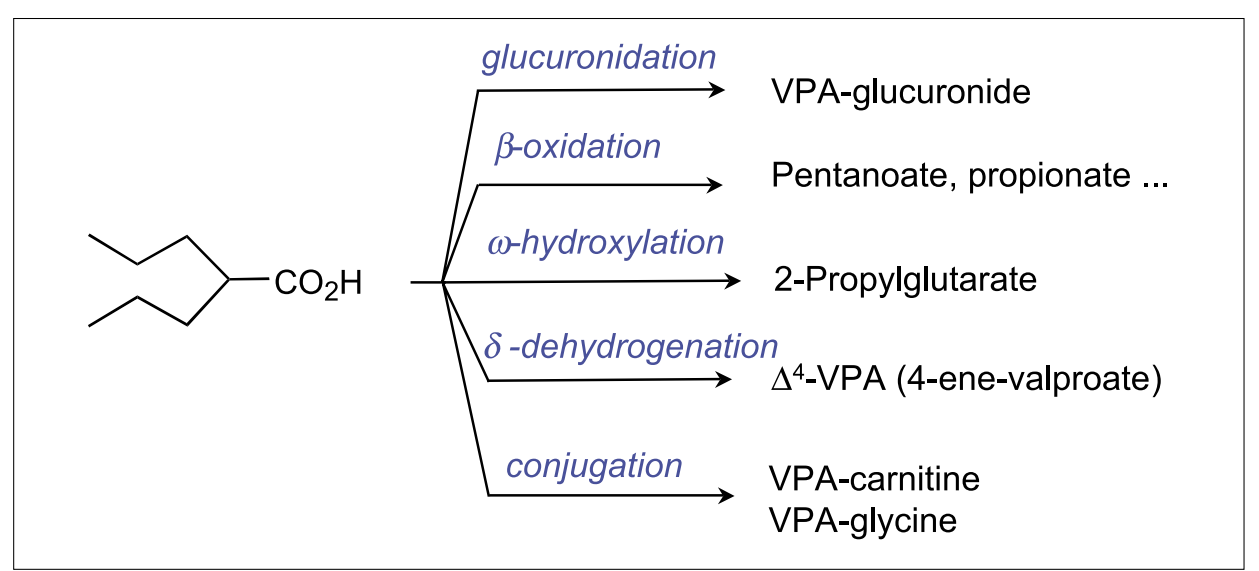

Fig. 2. Metabolism of valproate. Valproate is a medium-chain, branched fatty acid, which is primarily metabolized by glucuronidation and conjugation with carnitine or glycine. In addition, valproate can be degraded by $\beta$-oxidation, which produces odd-chain fatty acids such as propionate and pentanoate. It can also be metabolized to a $\omega$-hydroxy derivative, which can be converted to 4-enevalproate. These fatty acid derivatives and/or their corresponding CoA-esters can inhibit mitochondrial functions, in particular mitochondrial $\beta$-oxidation. 
side chain attached to the benzoyl moiety (Fig. 3, unpublished results). The composition of this side chain appears therefore to be critical for mitochondrial toxicity of amiodarone derivatives. Further studies will have to show whether these derivatives can block the hERG channels, and whether their activity to toxicity profile is favourable as compared to amiodarone.

\section{Benzbromarone and Bromarone}

Benzbromarone and bromarone are amiodarone derivatives (Fig. 3). For both substances, hepatotoxicity has been shown; in some patients with a fatal outcome [16]. Because of the structural similarity with amiodarone, we hypothesized that the toxicity of these two substances may also have a mitochondrial mechanism.

We could indeed show that both drugs decrease the mitochondrial membrane potential, inhibit the activity of the respiratory chain and uncouple oxidative phosphorylation. In addition, both drugs impaired mitochondrial $\beta$-oxidation [17].

Since inhibition of the electron transport chain can be associated with increased formation of oxygen radicals, we speculated that benzbromarone and benzarone could open the membrane permeability transition pore, leading to dissipation of the mitochondrial membrane potential and possibly initiating apoptosis. Indeed, we could demonstrate increased reactive oxygen species (ROS) formation in the presence of these substances, and opening of the membrane permeability transition pore; as well as mitochondrial swelling and cellular spillage of cytochrome c due to rupture of the outer mitochondrial membrane. This initiated either apoptosis or necrosis, depending on the cellular ATP content [17].

\section{Conclusions}

Many drugs can affect mitochondrial function, and can thereby lead to cellular and/or organ damage. Important mechanisms include inhibition of the respiratory chain, uncoupling of oxidative phosphorylation and inhibition of mitochondrial $\beta$-oxidation. These primary effects can secondarily lead to a decrease in the mitochondrial ATP content, increased production of ROS, and opening of the mitochondrial membrane permeability transition pore, with consecu-

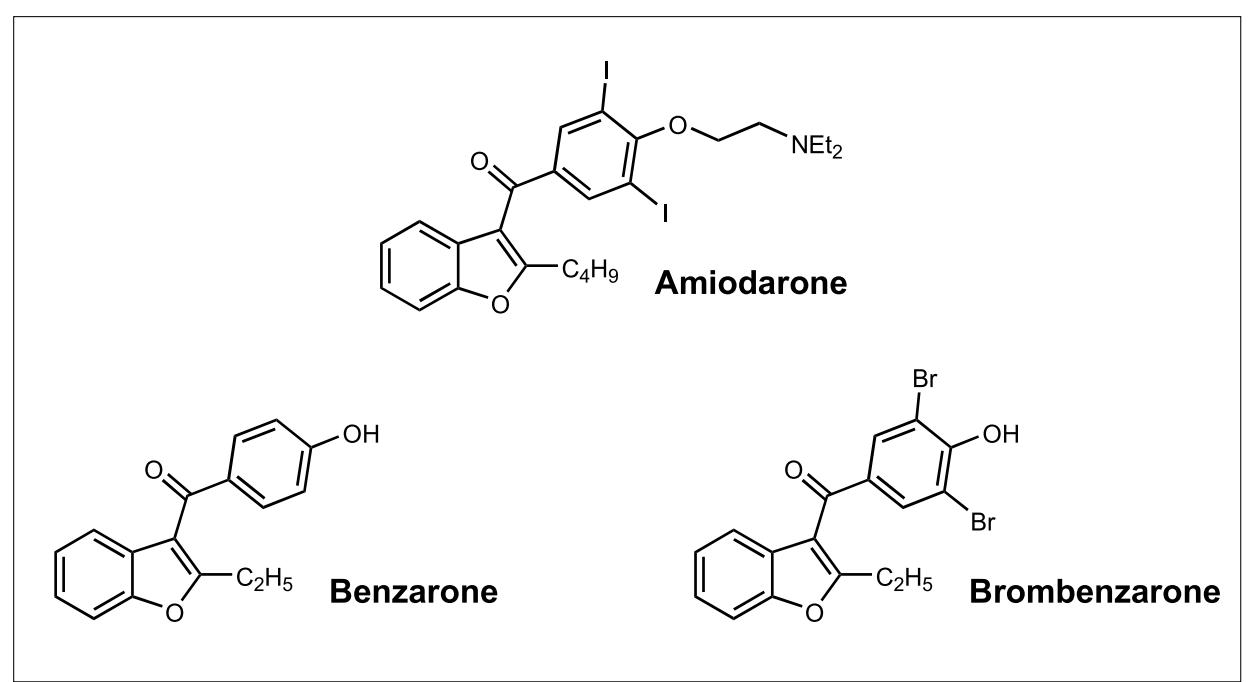

Fig. 3. Structure of amiodarone and amiodarone derivatives investigated. Amiodarone, benzbromarone and benzarone are 3-benzoyl-benzofuran derivatives. All of these three substances are mitochondrial toxins, mainly affecting the respiratory chain and $\beta$-oxidation. While amiodarone carries a diethylaminoethoxy side chain at the benzoyl moiety, this side chain is lacking for benzarone and benzbromarone. Alterations of this side chain, e.g. replacement by an ethoxy or acetoxy group, cause a reduction in the mitochondrial toxicity of amiodarone.

tive mitochondrial swelling, rupture of the outer membrane and cellular spillage of cytochrome c. These events can end in apoptosis or necrosis of the affected cells, depending on the cellular ATP content.

Received: January 16, 2006

[1] K.B. Wallace, A.A. Starkov, Annu. Rev. Pharmacol. Toxicol. 2000, 40, 353.

[2] B. Fromenty, D. Pessayre, Pharmacol. Ther. 1995, 67, 101.

[3] J.Y. Feng, A.A. Johnson, K.A. Johnson, K.S. Anderson, J. Biol. Chem. 2001, 276, 23832 .

[4] M. Le Bras, M.V. Clement, S. Pervaiz, C. Brenner, Histol. Histopathol. 2005, 20 , 205.

[5] F.E. Dreifuss, N. Santilli, D.H. Langer, K.P. Sweeney, K.A. Moline, K.B. Menander, Neurology 1987, 37, 379.

[6] S. Ponchaut, K. Veitch, Biochem. Pharmacol. 1993, 46, 199.

[7] S. Krahenbuhl, G. Mang, H. Kupferschmidt, P.J. Meier, M. Krause, Gut $\mathbf{1 9 9 5}$ 37, 140.

[8] S. Krahenbuhl, S. Brandner, S. Kleinle, S. Liechti, D. Straumann, Liver 2000, 20, 346.

[9] M.C. Laub, I. Paetzke-Brunner, G. Jaeger, Epilepsia 1986, 27, 559.
[10] L. Vernez, M. Wenk, S. Krahenbuhl, $R a-$ pid Commun. Mass Spectrom. 2004, 18, 1233.

[11] I. Tamai, R. Ohashi, J. Nezu, H. Yabuuchi, A. Oku, M. Shimane, Y. Sai, A. Tsuji, $J$. Biol. Chem. 1998, 273, 20378.

[12] J.H. Lewis, R.C. Ranard, A. Caruso, L.K. Jackson, F. Mullick, K.G. Ishak, L.B. Seeff, H.J. Zimmerman, Hepatology 1989 , 9, 679 .

[13] B. Fromenty, C. Fisch, G. Labbe, C. Degott, D. Deschamps, A. Berson, P. Letteron, D. Pessayre, J. Pharmacol. Exp. Ther. 1990, 255, 1371.

[14] B. Fromenty, C. Fisch, A. Berson, P. Letteron, D. Larrey, D. Pessayre, J. Pharmacol. Exp. Ther. 1990, 255, 1377.

[15] M. Spaniol, R. Bracher, H.R. Ha, F. Follath, S. Krahenbuhl, J. Hepatol. 2001, 35, 628.

[16] M.L. Hautekeete, J. Henrion, S. Naegels, A. DeNeve, M. Adler, C. Deprez, G. Devis, G. Kloppel, Liver 1995, 15, 25.

[17] P. Kaufmann, M. Torok, A. Hanni, P. Roberts, R. Gasser, S. Krahenbuhl, Hepatology 2005, 41, 925 . 